\title{
Interaction forces and molecular adhesion between pre-adsorbed poly(ethylene imine) layers
}

\author{
Ramon Pericet-Camara $^{\mathrm{a}}$, Georg Papastavrou ${ }^{\mathrm{a}, *}$, Sven H. Behrens ${ }^{\mathrm{b}}$, Christiane A. Helm ${ }^{\mathrm{c}}$, \\ Michal Borkovec ${ }^{\text {a }}$ \\ ${ }^{a}$ Laboratory of Colloid and Surface Chemistry, Department of Inorganic, Analytical, and Applied Chemistry, University of Geneva, Sciences II, \\ 30 Quai Ernest Ansermet, 1211 Geneva 4, Switzerland \\ b Polymer Research Laboratory, BASF Aktiengesellschaft, Ludwigshafen, Germany \\ ${ }^{\mathrm{c}}$ Institute of Physics, Applied Physics, Ernst-Moritz-Arndt University, Friedrich-Ludwig-Jahn-Str. 16, 17489 Greifswald, Germany
}

Received 19 July 2005; accepted 15 September 2005

Available online 21 October 2005

\begin{abstract}
Interaction forces between pre-adsorbed layers of branched poly(ethylene imine) (PEI) of different molecular mass were studied with the colloidal probe technique, which is based on atomic force microscopy (AFM). During approach, the long-ranged forces between the surfaces are repulsive due to overlap of diffuse layers down to distances of a few nanometers, whereby regulation of the surface charge is observed. The ionic strength dependence of the observed diffuse layer potentials can be rationalized with a surface charge of $2.3 \mathrm{mC} / \mathrm{m}^{2}$. The forces remain repulsive down to contact, likely due to electro-steric interactions between the PEI layers. These electro-steric forces have a range of a few nanometers and appear to be superposed to the force originating from the overlap of diffuse layers. During retraction of the surfaces, erratic attractive forces are observed due to molecular adhesion events (i.e., bridging adhesion). The frequency of the molecular adhesion events increases with increasing the ionic strength. The force response of the PEI segments is dominated by rubber-like extension profiles. Strong adhesion forces are observed for low molecular mass PEI at short distances directly after separation, while for high molecular mass weaker adhesion forces at larger distances are more common. The work of adhesion was estimated by integrating the retraction force profiles, and it was found to increase with the ionic strength. (c) 2005 Elsevier Inc. All rights reserved.
\end{abstract}

Keywords: Surface forces; Branched polyelectrolytes; Adhesion; Colloidal probe

\section{Introduction}

Poly(ethylene imine) (PEI) is a cationic branched weak polyelectrolyte, which strongly adsorbs on negatively charged surfaces, and thereby leads to their charge reversal. It is used in a wide variety of industrial applications, for example, as stabilizer of colloidal slurries, as flocculant in water purification, or as retention aid in papermaking [1-4]. Besides these classical applications, PEI has gained importance in the design of flat or spherical polyelectrolyte multilayers [5-8]. Their permeability and surface functionality can be controlled by judicious choice of their building blocks, and such systems are therefore becoming interesting for drug delivery, cosmetics, or in situ

\footnotetext{
* Corresponding author.

E-mail address: georg.papastavrou@unige.ch (G. Papastavrou).
}

sensing [6,9]. Multilayers are prepared by alternating adsorption of two oppositely charged polyelectrolytes on a suitable substrate, whereby PEI is often used as the precursor layer [7,8]. In between the adsorption steps, the substrate is washed in a polyelectrolyte-free solution, and thus such pre-adsorbed polyelectrolyte films are no longer in contact with a bulk solution of the polyelectrolyte in question. This situation is different to many of the applications mentioned above, where the surface is always in contact with a polyelectrolyte solution.

The adsorption of PEI to water-solid interfaces has been examined by adsorption experiments [2,10], electrokinetic methods $[11,12]$, or optical reflectometry $[13,14]$. Besides these classical techniques, direct force measurements with the surface force apparatus (SFA) or the atomic force microscope (AFM) have substantially contributed to our understanding of the interactions between adsorbed polyelectrolyte films [14-17]. In- 
teraction forces between mica or glass surfaces in PEI solutions have been systematically examined with the SFA or its non-interferometric counterpart, which is commonly referred to as MASIF (i.e., measurement and analysis of surface interaction forces) $[16,17]$. These studies conclude that adsorbed PEI leads to long-range electrostatic interactions, whose magnitude increases with increasing PEI concentration [16]. The pull-off forces decrease with increasing PEI concentration and thus follow an opposite trend to the long-range forces [17]. Preadsorbed films of PEI were investigated in lesser detail, primarily with respect to polyelectrolyte multilayer build-up $[8,18]$, or as base for the covalent coupling with further polymers [14].

In this study, we use the colloidal probe technique to measure the interaction forces between pre-adsorbed PEI layers on quartz. The colloidal probe technique is based on the atomic force microscope (AFM) and uses a cantilever with a colloidal particle attached to its end [19-21]. This technique has the advantage of well-defined interaction geometry, as the SFA or the MASIF. Due to the use of an AFM-cantilever with a low spring constant, the colloidal probe technique is also sensitive to the bridging of single polymers during the separation of probe and sample [22-25]. Thus, this technique is ideally suited to study the long-range interactions as well as the adhesion forces between adsorbed polyelectrolyte layers.

The adsorption conditions of the PEI layers were chosen to mimic the PEI-precursor layer used for the preparation of polyelectrolyte multilayers [7,26-28]. With respect to longrange interaction forces, we studied the charge regulation of the weak polyelectrolyte PEI layers for the first time, and examined the effect of the molecular mass. The bridging adhesion between pre-coated PEI layers was measured for different ionic strengths, and examined on the level of single detachment events.

\section{Experimental section}

\subsection{Pre-adsorbed layers and AFM imaging}

Fractions of highly branched poly(ethylene imine) (PEI) with molecular masses of approximately 2, 30, 300, and $5000 \mathrm{kDa}$ were used. The first one was purchased from SigmaAldrich, and all other PEI fractions were isolated in the BASF laboratories (Ludwigshafen, Germany). The aqueous solutions of $33-50 \%$ weight percent of the polymers were used without further purification. The degree of ionization of PEI is about $70-80 \%$ at $\mathrm{pH} 4$ in aqueous solution [29]. All solutions were prepared with Milli-Q grade water (Millipore).

Polished quartz plates (Schott SA, Switzerland) were used as substrates for PEI adsorption in order to mimic the surface of the silica spheres used as colloidal probes. The substrates were initially treated with $65 \%$ nitric acid at $95^{\circ} \mathrm{C}$, subsequently with a 1:4 hot mixture of $\mathrm{H}_{2} \mathrm{O}_{2}(35 \%)$ and $\mathrm{H}_{2} \mathrm{SO}_{4}$, and finally rinsed extensively with Milli-Q water. Pre-adsorbed PEI layers were prepared by immersing the substrates in a PEI solution with a concentration of $200 \mathrm{mg} / \mathrm{L}$ at $\mathrm{pH} 9.5$ and a $\mathrm{KCl}$ concentration of $0.1 \mathrm{M}$ during $12 \mathrm{~h}$ at room temperature (high salt conditions). For comparison, the PEI layers were prepared in a PEI solu- tion of the same concentration at $\mathrm{pH} 9.8$ without any added salt (low salt conditions). Afterwards, the surfaces were thoroughly rinsed with Milli-Q water.

The bare quartz substrates or the pre-adsorbed layers were imaged in dry state with a Nanoscope IIIa AFM (Veeco) in the tapping mode. Better resolution for AFM imaging was obtained when naturally oxidized silicon wafers (Silchem, Freiburg, Germany) were used as substrates. The wafers were cleaned with the RCA method, which consists of incubating the wafer in a 1:1:5 mixture of hydrogen peroxide $(35 \%)$, ammonia $(25 \%)$, and water at $75^{\circ} \mathrm{C}$ for $10 \mathrm{~min}[30]$.

\subsection{Direct force measurements}

The colloidal probe technique was used for the force measurements with a closed-loop AFM (MFP-3D, Asylum Research, Santa Barbara, CA). Colloidal probes were prepared with colloidal silica particles (Bangs Laboratories) with a diameter in the range of 6-7 $\mu \mathrm{m}$. Their actual diameters were determined with optical microscopy to an accuracy of about $0.3 \mu \mathrm{m}$. The particles were attached to the end of a tip-less AFM-cantilever (CSC 12, $\mu$-masch, Estonia) with UV-curable glue (NO 63, Norland Adhesives) utilizing a dedicated micromanipulator (Märzhäuser, Germany). The glue was cured by illuminating the probe with the mercury lamp in the optical microscope for about one minute. The spring constants of the AFM-cantilevers were determined by analyzing their thermal fluctuations in air $[31,32]$. The results were compared with values obtained from the procedure proposed by Sader et al. [33], which uses the frequency response of the cantilever, its topview geometrical dimensions, and the properties of the surrounding medium. The results of both methods deviated by less than $25 \%$ in all cases.

Prior to the adsorption experiments, the colloidal probes and the quartz substrates were cleaned in air-plasma at $35 \mathrm{~W}$ for 5 min (Harrick Scientific, NY). The substrates were fixed with the UV-curable glue to the bottom of petri dishes, which served as open AFM fluid cells. The pre-adsorbed PEI films on the substrates and the probes were prepared as described in Section 2.1. The petri dish was subsequently filled with a $\mathrm{KCl}$ solution of desired ionic strength, which was previously adjusted to $\mathrm{pH} 4$ with dilute $\mathrm{HCl}$. The $\mathrm{pH}$ was checked before and after each measurement with a microelectrode. The force curves were acquired at a cycling frequency of $0.3 \mathrm{~Hz}$ resulting in approach and retraction velocities of $0.2-0.6 \mu \mathrm{m} / \mathrm{s}$. Force distance curves were measured at three different positions on the substrate, and 50 force curves were measured for each position. All measurements were carried out at room temperature of $23 \pm 2{ }^{\circ} \mathrm{C}$.

The approximate symmetry of the system consisting of a colloidal silica particle and a quartz substrate in respect to the electrostatic interactions was verified by yet unpublished force measurements in the sphere-sphere geometry. For this purpose, a colloidal silica sphere was glued to a solid substrate, while another silica sphere was used as colloidal probe. These measurements were compared with the ones obtained on a quartz surface and were found to be equal for the bare surfaces within experimental error. We conclude from these measurements that 
also the PEI-covered surfaces are approximately symmetric in respect to their electrostatic interactions.

\subsection{Force data analysis}

Force versus distance curves were calculated from the cantilever deflection and the piezo-crystal displacement. The force $F$ was determined from the deflection and the spring constant with a sensitivity of about $20 \mathrm{pN}$. The separation distance $D$ is obtained from the displacement of the piezo-crystal and by determining the zero separation from a linear fit of the constant compliance region. In the present case, this point can be determined with an accuracy of about $0.5 \mathrm{~nm}$, since the pre-adsorbed polyelectrolyte layers are sufficiently thin and become incompressible under load. Further details on this technique can be found in the literature $[34,35]$.

The interaction forces upon approach were obtained by averaging about 50 force curves measured at the same location, and normalized by the probe radius $R$. Derjaguin's approximation suggests that the resulting normalized force $F / R$ is proportional to the free energy of interaction per unit area [36]. The force versus distance profiles were fitted to the full numerical solution of the Poisson-Boltzmann equation for two symmetric plates for distances above $\kappa^{-1} / 2$ for ionic strengths $<10 \mathrm{mM}$ and $\kappa^{-1}$ for $\geqslant 10 \mathrm{mM}$ [37]. When the parameters entering the constant regulation (CR) model are determined in this fashion, one can accurately predict the force at smaller distances down to a few nanometers.

The surface charge was assumed to be localized in a plane positioned at zero separation as obtained from the constant compliance region, whereby the extension of the polymer layer was not taken into account [38]. In general, van der Waals forces were not considered, except for comparison. In the latter case, the plane of origin of the van der Waals force was displaced by a distance $d$, whereby $d>0$ corresponds to a shift towards the solid substrate.

Molecular adhesion events were identified from the retraction force curves by an automated procedure based on an algorithm for the detection of local minima in the force profile [39]. In order to classify a minimum in the force as an adhesion event, the slope within the jump-off instability had to agree with the spring constant of the cantilever within 50\%, and the magnitude of the force prior to the jump-off had to exceed the noise in the non-contact region by a factor of 5 . The work of adhesion was evaluated from the area between approach and redraw parts, the force curves were smoothed with piece-wise parabolic fits and integrated with the trapezoidal rule.

\section{Results and discussion}

Direct force measurements between pre-adsorbed layers of branched poly(ethylene imine) (PEI) on silica substrates were carried out in $\mathrm{KCl}$ solutions at $\mathrm{pH} 4$ with the colloidal probe technique. The influence of salt concentration and molecular mass of PEI on the interaction forces was investigated in detail. The PEI layers were adsorbed at $\mathrm{pH} 9.5-9.8$ at a concentration of $200 \mathrm{mg} / \mathrm{L}$ and two different salt concentrations, in order to
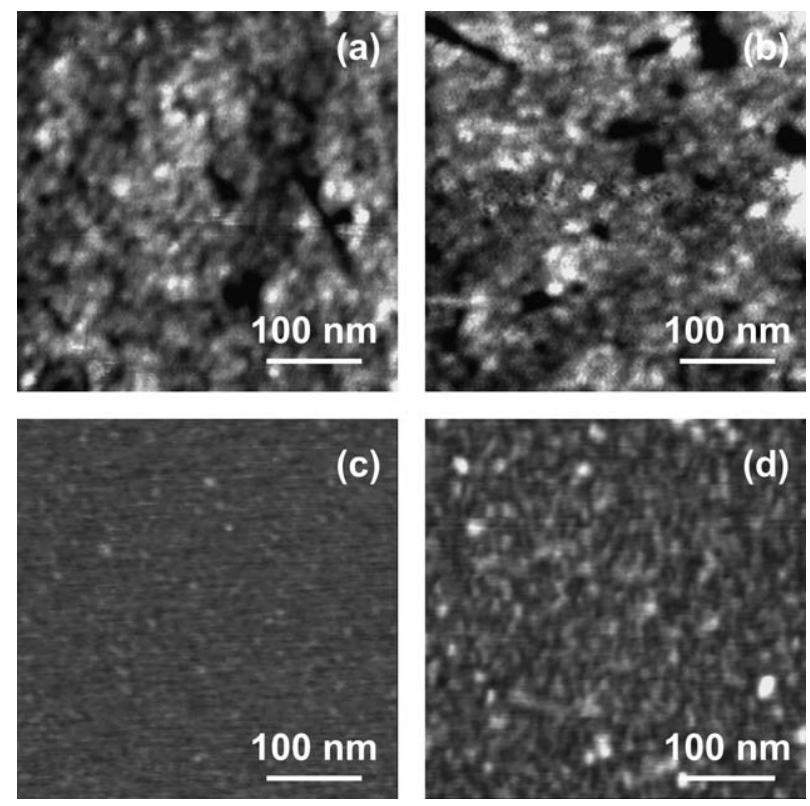

Fig. 1. Surface topography for pre-coated poly(ethylene imine) (PEI) films by tapping mode AFM. (a) Bare quartz substrate and (b) 5 MDa PEI on quartz. Oxidized silicon wafers with pre-adsorbed PEI of (c) $2 \mathrm{kDa}$ and (d) $5 \mathrm{MDa}$.

mimic conditions for the preparation of pre-cursor layers for multilayers [7,26-28]. In the following, we shall focus on PEI layers adsorbed at high salt conditions. For the low salt adsorption conditions, similar results were obtained.

The surface topography of the pre-adsorbed PEI layers is shown in Fig. 1. The layers are relatively uniform and homogeneous. The quartz substrates show few scratches and irregularities, and the flat parts of the surface are relatively rough with a root mean square (RMS) roughness of about $0.3 \mathrm{~nm}$ (Fig. 1a). Even the PEI of high molecular mass $(5000 \mathrm{kDa})$ cannot be reliably identified on such substrates, and upon its adsorption the roughness of the substrates remains basically unchanged (Fig. 1b). For this reason, we have imaged the pre-adsorbed PEI layers on oxidized silicon wafers, which were much more smooth over large areas with RMS roughness of $0.16 \mathrm{~nm}$. Adsorbed PEI of low molecular mass $(2 \mathrm{kDa})$ forms very homogeneous layers with RMS roughness of $0.06 \mathrm{~nm}$ (Fig. 1c). The fact that this roughness is smaller than the roughness of the bare silicon wafers suggests that the PEI adsorbs in the dints on the surface, and thereby flattens the substrate. For the adsorbed PEI of the high molecular mass (5000 kDa), the RMS roughness increases to $0.15 \mathrm{~nm}$ and becomes comparable to the roughness of the bare substrate (Fig. 1d). The characteristic length scale of the lateral surface heterogeneities is on the order of 20-50 $\mathrm{nm}$. These heterogeneities probably correspond to individual adsorbed PEI molecules, which show almost a closed packed structure on the surface, but leaving small patches of uncovered substrate in between. The lateral extent of individual PEI molecules with a molecular mass of $1000 \mathrm{kDa}$ adsorbed on mica was reported around $60 \mathrm{~nm}$ with an average height of mere $1.5 \mathrm{~nm}$ [40]. The reported flat pancake-like structure of adsorbed PEI results primarily from the strong electrostatic attraction between the positively charged polyelectrolyte and the 

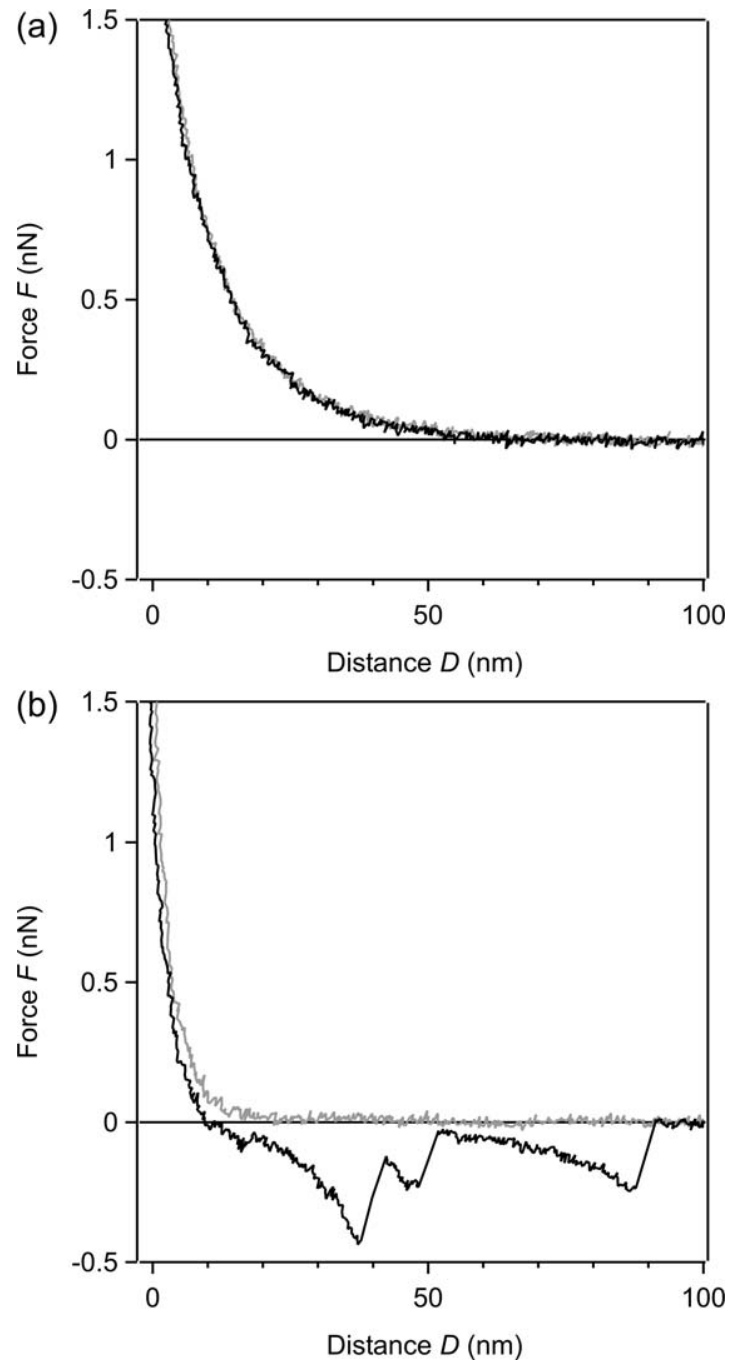

Fig. 2. Representative force profiles between colloidal silica probe and quartz substrate with pre-adsorbed layers of $5000 \mathrm{kDa}$ PEI prepared at high salt adsorption conditions. The approach (grey) and retraction (black) force curves were measured at $\mathrm{pH} 4$ and different ionic strengths in $\mathrm{KCl}$. (a) Reversible force curve at $1 \mathrm{mM}$ and (b) molecular adhesion events upon retraction at $100 \mathrm{mM}$.

negatively charged substrate. A similar adsorption mechanism is likely to be operational in the present situation, and will result in pre-adsorbed layers, which correspond to monolayers with a thickness of few nanometers only.

The relevant features of the interaction forces between such pre-adsorbed PEI layers are summarized in Fig. 2. In order to ensure a symmetric system, the measurements were carried out with a silica probe against a quartz surface. For all conditions investigated, the forces between the surfaces are repulsive upon approach. At low ionic strengths, these forces are long-ranged and always repulsive, upon approach as well as upon retraction (Fig. 2a). At higher ionic strengths, the repulsive part is shorter ranged, and upon retraction one frequently observes molecular adhesion events (Fig. 2b). The erratic adhesion events occur due to bridging of PEI molecules at contact and their subsequent pulling upon retraction. Due to the branched architecture of the PEI, however, they cannot be easily decomposed into contributions from individual molecules. In the following, we shall first quantitatively analyze the repulsive forces, and later discuss the nature of the molecular adhesion events along with a statistical analysis.

We found no difference between forces measured for freshly prepared substrates and for substrates, which were stored in a solution of $\mathrm{pH} 4$ for several hours. These findings confirm the good stability of pre-adsorbed PEI films in contact with electrolyte solutions, and they are in full accord with similar conclusions based on optical reflectivity [13].

\subsection{Repulsive forces upon approach}

The repulsive force profiles measured upon approach can be quantitatively interpreted in terms of interactions between charged surfaces across an electrolyte solution. The experimental force data, which were normalized to the probe radius, were interpreted in terms of the exact solution of the non-linear Poisson-Boltzmann (PB) equation for interacting, symmetric plates, including constant charge (CC), constant potential (CP), and constant regulation (CR) boundary conditions [37]. All three solutions coincide at larger distances. In this region, they only depend on the diffuse layer potential $\psi_{d}$ and the Debye length

$\kappa^{-1}=\sqrt{\frac{\varepsilon \varepsilon_{0} k T}{2 N_{A} e^{2} I}}$,

where $\varepsilon \varepsilon_{0}$ the total permittivity of water, $k T$ the thermal energy, $N_{A}$ the Avogadro's number, $e$ the elementary charge, and $I$ the ionic strength of the solution. At shorter distances, boundary conditions on the surface become important, since the surface charge may regulate upon approach due to adsorption of charged species (e.g., protons, salt ions). The simplest way to consider this effect is the recently proposed constant regulation (CR) boundary condition [37]. This approximation stipulates a linear response of the surface charge to the diffuse layer potential, and characterizes the ability of the surface to regulate its charge by introducing a regulation parameter $p$. For a surface that does not modify its charge (CC), we have $p=1$, while for a surface that regulates its charge easily (CP), we have $p=0$. For a realistic surface, the regulation parameter $p$ usually lies in between these two limits.

Let us first discuss the interpretation of a typical forcedistance curve in terms of the PB model without considering van der Waals forces. The latter will be shown to be negligible in the next paragraph. Fig. 3a compares illustrative experimental data points measured at an ionic strength of $1 \mathrm{mM}$ for $2 \mathrm{kDa}$ PEI with model calculations. The employed PB model assumes that the point of contact between the surfaces coincides with the plane of origin of the electrostatic force. While neither CC nor $\mathrm{CP}$ conditions are capable of describing the experimental data correctly, the CR model fits the data very well almost down to contact. The fitted parameters extracted from the data are the Debye length $\kappa^{-1}=8.7 \mathrm{~nm}$, the diffuse layer potential $\psi_{d}=32 \mathrm{mV}$, and the regulation parameter $p=0.69$. These parameters are also summarized in Table 1 . Based on the ionic strength of $1 \mathrm{mM}$, the expected Debye length is $9.6 \mathrm{~nm}$, which 

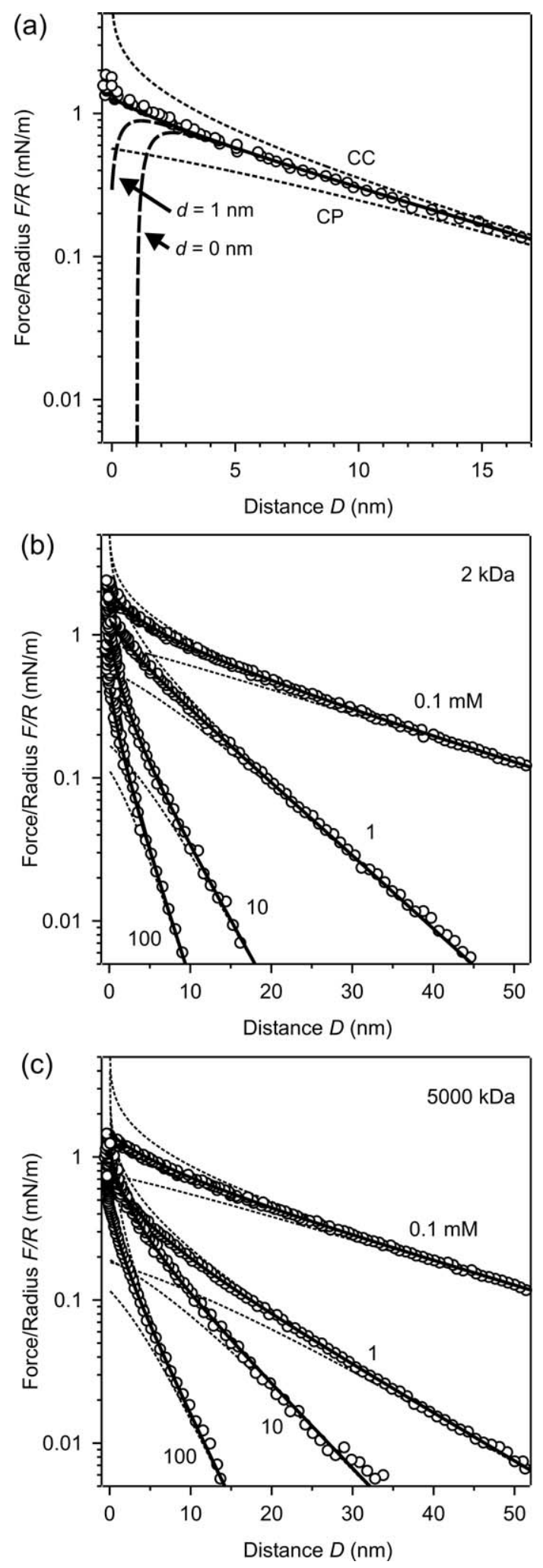

Fig. 3. Force profiles measured upon approach between pre-adsorbed PEI layers on silica prepared at high salt conditions and measured at $\mathrm{pH} 4$ and different ionic strengths. Lines are best fits with the PB model with constant charge (CC, dotted curve top), constant potential (CP, dotted curve bottom), and constant regulation (CR, solid line). (a) Detail of the force profile for $2 \mathrm{kDa}$ PEI at $1 \mathrm{mM}$ whereby the effect of the van der Waals force and relative shift of the planes of origin $d$ are shown (dashed). Force profiles at different ionic strengths with PEI of molecular mass (b) 2 and (c) $5000 \mathrm{kDa}$.
Table 1

Poisson-Boltzmann (PB) model parameters obtained from direct force measurements at $\mathrm{pH} 4$

\begin{tabular}{|c|c|c|c|c|c|c|c|}
\hline \multirow[t]{2}{*}{$\begin{array}{l}\text { Molecular } \\
\text { mass } \\
(\mathrm{kDa})\end{array}$} & \multirow[t]{2}{*}{$\begin{array}{l}\text { Ionic } \\
\text { strength } \\
I(\mathrm{mM})\end{array}$} & \multicolumn{2}{|c|}{$\begin{array}{l}\text { Debye } \\
\text { length } \\
\kappa^{-1}(\mathrm{~nm})\end{array}$} & \multicolumn{2}{|c|}{$\begin{array}{l}\text { Diffuse layer } \\
\text { potential } \\
\psi_{d}(\mathrm{mV})\end{array}$} & \multicolumn{2}{|c|}{$\begin{array}{l}\text { Regulation } \\
\text { parameter } \\
p\end{array}$} \\
\hline & & High $^{\mathrm{a}}$ & Low $^{\mathrm{b}}$ & $\mathrm{High}^{\mathrm{a}}$ & Low $^{b}$ & $\mathrm{High}^{\mathrm{a}}$ & Low $^{b}$ \\
\hline \multirow[t]{4}{*}{2} & 0.1 & $22.1^{\mathrm{d}}$ & 24.6 & $58.2^{\mathrm{d}}$ & 72.4 & $0.69^{d}$ & 0.63 \\
\hline & 1 & $8.7^{\mathrm{c}, \mathrm{d}}$ & 9.0 & $31.6^{\mathrm{c}, \mathrm{d}}$ & 27.9 & $0.69^{\mathrm{c}, \mathrm{d}}$ & 0.67 \\
\hline & 10 & $4.3^{\mathrm{d}}$ & 3.5 & $15.2^{\mathrm{d}}$ & 18.9 & $0.76^{\mathrm{d}}$ & - \\
\hline & 100 & $2.9^{\mathrm{d}}$ & - & $7.4^{\mathrm{d}}$ & - & $0.78^{d}$ & - \\
\hline \multirow[t]{4}{*}{30} & 0.1 & _- & 34.1 & - & 83.0 & - & 0.76 \\
\hline & 1 & 8.7 & 9.6 & 23.2 & 24.0 & 0.72 & 0.78 \\
\hline & 10 & 5.1 & 5.9 & 13.2 & 12.6 & 0.77 & 0.85 \\
\hline & 100 & 2.0 & - & 6.9 & - & 0.71 & - \\
\hline \multirow[t]{4}{*}{300} & 0.1 & - & 26.6 & - & 66.8 & - & 0.67 \\
\hline & 1 & 10.9 & 8.6 & 30.3 & 24.7 & 0.84 & 0.69 \\
\hline & 10 & 5.8 & 4.8 & 25.5 & 13.0 & 0.60 & 0.72 \\
\hline & 100 & 1.5 & - & 9.1 & - & 0.42 & - \\
\hline \multirow[t]{4}{*}{5000} & 0.1 & $23.5^{\mathrm{e}}$ & 25.8 & $58.7^{\mathrm{e}}$ & 101.9 & $0.58^{\mathrm{e}}$ & 0.42 \\
\hline & 1 & $13.5^{\mathrm{e}}$ & 9.0 & $24.7^{\mathrm{e}}$ & 41.1 & $0.79^{\mathrm{e}}$ & 0.65 \\
\hline & 10 & $7.0^{\mathrm{e}}$ & 4.2 & $17.9^{\mathrm{e}}$ & 16.9 & $0.79^{\mathrm{e}}$ & 0.86 \\
\hline & 100 & $3.6^{\mathrm{e}}$ & - & $10.7^{\mathrm{e}}$ & - & $0.64^{\mathrm{e}}$ & - \\
\hline
\end{tabular}

a High salt (100 mM at $\mathrm{pH} 9.5)$ adsorption conditions for PEI.

b Low salt (no salt added at $\mathrm{pH} 9.8$ ) adsorption conditions for PEI.

${ }^{c}$ Fig. 3a, d Fig. 3b, and e Fig. 3c show the corresponding curves.

is reasonably close to the decay length extracted from the fit. These values were obtained from a fit at larger distances (see Section 2). Fits over the whole distance range lead to very similar parameters.

The existence of very short-ranged repulsion and the absence of jump-in instabilities indicate that short-ranged electro-steric repulsion overrides the attractive van der Waals forces. Similar trends were already reported for interactions between adsorbed PEI layers as measured by MASIF or the colloidal probe technique $[8,14,17]$. Fig. 3 a shows clearly that the experimental data are inconsistent with the assumption that the planes of origin for the van der Waals force and of the diffuse layer coincide $(d=0)$. We have used a Hamaker constant of $8.3 \times 10^{-21} \mathrm{~J}$ as appropriate for quartz [36], and neglected the contribution of the polymer layer. Agreement with the experimental force profiles can be obtained by displacing the plane of origin of the van der Waals force towards the solid substrate by at least $1 \mathrm{~nm}$ ( $d>0$, Fig. 3a). This shift can be interpreted in terms of the finite thickness of the polymer layer, which gives only a negligible contribution to the van der Waals force. The Hamaker constant of the layer will be close to the one of water, since the water content of the layer is relatively high. For these reasons, neither van der Waals forces nor electro-steric forces were taken into account in the data analysis.

The results of the force measurements between pre-adsorbed layers prepared at high salt with PEI of low and high molecular mass are summarized in Figs. $3 b$ and $3 c$ for different ionic strengths. The force versus distance curve can be well described by the solutions of the PB equation with CR boundary conditions, and deviations from this model are only observable for very small separation distances below approximately 
$2 \mathrm{~nm}$. For the two extreme molecular masses there are no significant differences between these results. The fitted parameters are summarized in Table 1 and Fig. 4, along with very similar results from the pre-adsorbed layers prepared at low salt. We did not find significant differences for the interaction forces between pre-adsorbed layers when the pure electrolyte solution $(1 \mathrm{mM} \mathrm{KCl}, \mathrm{pH} 4)$ was replaced by solution of the same background electrolyte concentration and $\mathrm{pH}$ but contained additionally $50 \mathrm{mg} / \mathrm{L}$ PEI ( $30 \mathrm{kDa})$.

Fig. 4a shows that the fitted Debye lengths are always in good agreement with the ones expected from the ionic strength of the solution at sufficiently low ionic strengths (Eq. (1)). Disagreement is observed at high ionic strength, whereby deviations are noticeable at $10 \mathrm{mM}$, and they are very pronounced at $100 \mathrm{mM}$. In the latter case, range of the interactions is small, but larger than expected on basis of the PB model. While its exponential dependence can be described with this model reasonably well, the observed averaged decay lengths of $2.9 \pm 0.9 \mathrm{~nm}$ exceed significantly the expected Debye length of $0.9 \mathrm{~nm}$. While no systematic trend with the molecular mass is observed, we suspect that this repulsive force originates from the shortranged electro-steric repulsion between PEI layers. The relative independence of the electro-steric forces on the molecular mass suggests a flat configuration for the pre-adsorbed PEI molecules. This electro-steric force also prevents the jump-in of the surfaces at short distances, similar to steric repulsion between adsorbed polymer layers in good solvents [36].

Similar decay lengths to the ones reported here were derived from interaction forces between PEI layers in $50 \mathrm{mM} \mathrm{NaCl}$ near neutral $\mathrm{pH}$ [14]. These authors report decay lengths in the range of $2.1 \pm 0.3 \mathrm{~nm}$, which in their case also exceed the expected Debye length of $1.4 \mathrm{~nm}$. These decay lengths are comparable to the thickness of individual adsorbed PEI molecules [40].

The diffuse layer potentials $\psi_{d}$ are shown as a function of the ionic strength in Fig. 4b. In spite of the fact that we cannot determine the sign of the potential, we are confident that the adsorbed PEI leads to a charge reversal and that the surfaces are positively charged. The clearest evidence for this charge reversal stems from electrokinetic data $[11,12]$. The presently measured diffuse layer potential increases with decreasing ionic strength, and features a marginal dependence on the molecular mass and preparation conditions. The only exception are the pre-adsorbed layers prepared with the PEI of highest molecular mass of $5000 \mathrm{kDa}$, as they show somewhat higher surface potentials, particularly when prepared at low salt adsorption conditions. The diffuse layer potentials at $0.1 \mathrm{mM}$ reported here agree reasonably well with values based on force measurements between similar PEI layers, which were around $+60 \mathrm{mV}$ when measured with the colloidal probe [14] and about $+90 \mathrm{mV}$ with MASIF [17]. However, these measurements cannot be directly compared with our findings, since the mentioned studies used higher $\mathrm{pH}$ and lower ionic strengths. Electrokinetic data of adsorbed PEI on silica at concentrations of $50 \mathrm{mg} / \mathrm{L}$ indicate a strong dependence of the surface potential as a function of $\mathrm{pH}$ [11]. At $\mathrm{pH} 4$, the streaming potentials of oxidized silicon wafers in the presence of PEI are reported near +30 and $+55 \mathrm{mV}$ at ionic strengths of 10 and $1 \mathrm{mM}$, respectively. These
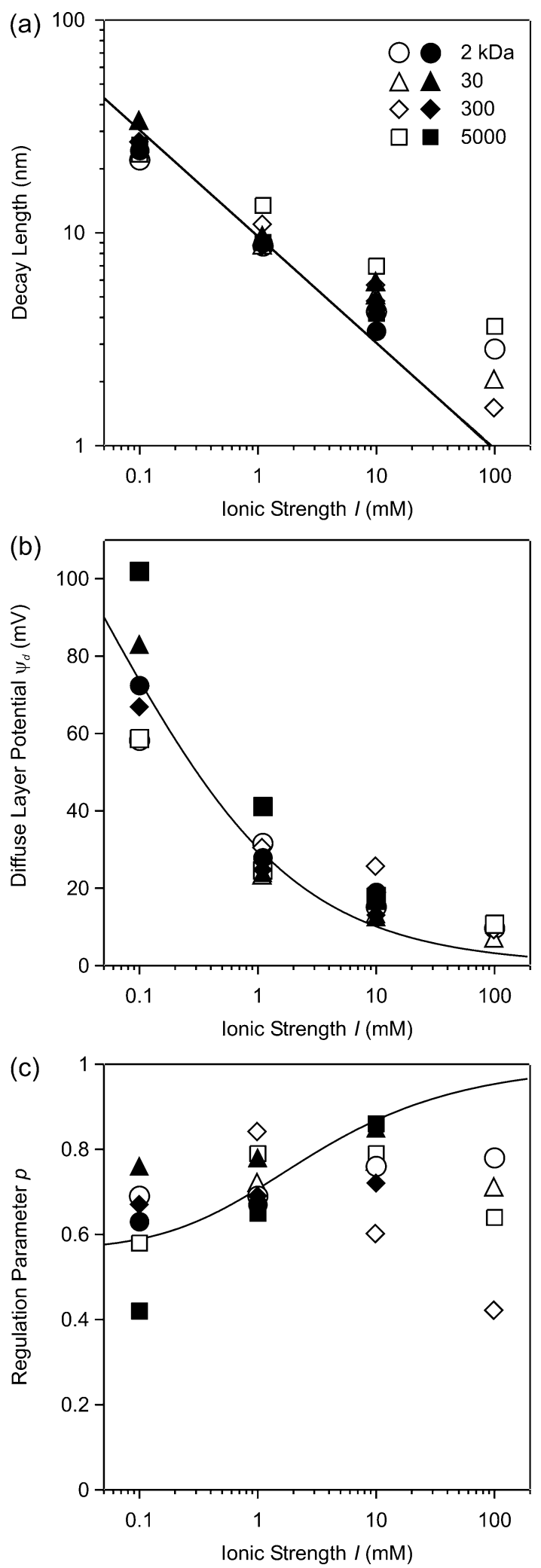

Fig. 4. Parameters resulting from the PB model and the constant regulation (CR) approximation as a function of the ionic strength for PEI layers pre-adsorbed at low (full symbols) and high (open symbols) salt conditions. The solid line indicates predictions of the PB theory. (a) Debye length (cf. Eq. (1)), (b) diffuse layer potential from the Grahame equation with a charge density of $2.3 \mathrm{mC} / \mathrm{m}^{2}$ (cf. Eq. (2)), and (c) regulation parameter with the inner capacitance of $36 \mathrm{mF} / \mathrm{m}^{2}$ (cf. Eqs. (3) and (4)). 
values are in reasonable agreement with the presently measured values, and the small discrepancies could be related to the uncertainties in positioning the plane of shear.

The ionic strength dependence of the diffuse layer potentials can well be described with the inverse Grahame equation

$\psi_{d}=\frac{2 k T}{e} \operatorname{asinh}\left(\frac{e \sigma}{2 k T \varepsilon \varepsilon_{0} \kappa}\right)$,

where the diffuse layer potential is expressed as a function of the surface charge and the ionic strength, which enters through the Debye length $\kappa^{-1}$ (cf. Eq. (1)). As shown in Fig. 4b, the Grahame equation describes the ionic strength dependence with a surface change density $\sigma=2.3 \mathrm{mC} / \mathrm{m}^{2}$ rather well. While the adsorbed PEI leads to charge reversal, it only corresponds to one positive charge per $70 \mathrm{~nm}^{2}$. This value indicates that only a small fraction of the PEI charge contributes to the diffuse layer charge, and that the PEI is neutralized by both, the silica surface and the bound counterions. The fact that all data are consistent with the same value of the surface charge represents a strong indication that the adsorbed amount of PEI is independent of the molecular mass, and is in accord with the weak molecular mass dependence of the adsorbed amount of PEI on colloidal silica particles $[2,10]$.

The regulation parameter obtained from the fits is plotted in Fig. $4 \mathrm{c}$ as a function of the ionic strength. The values lie mostly in the range of 0.6-0.8. At low ionic strength, the parameter increases with the ionic strength. This trend can be qualitatively understood since the diffuse layer capacitance increases with increasing ionic strength, while the capacitance of the inner layer remains approximately constant. One can attempt to quantify this trend with the relation proposed earlier [41]

$p=\frac{C_{d}}{C_{d}+C_{i}}$,

where $C_{i}$ is the inner layer capacitance, and the diffuse layer capacitance is given by

$C_{d}=\varepsilon \varepsilon_{0} \kappa \cosh \left(\frac{e \psi_{d}}{2 k T}\right)$.

As shown in Fig. 4c, the trend in the experimental data can be approximately reproduced with an inner layer capacitance of $C_{i} \simeq 36 \mathrm{mF} / \mathrm{m}^{2}$. However, at higher ionic strengths, the regulation parameter shows a strong scatter and decreases with increasing the ionic strength. These features indicate that the PB model is inappropriate to describe the interactions at short distances, and provides further evidence that the nature of the interactions is modified due to the presence of electro-steric forces. In general, we observe very similar trends for pre-adsorbed layers prepared at high salt and low salt adsorption conditions (see Table 1).

\subsection{Molecular adhesion events upon retraction}

We have already mentioned the molecular adhesion events observed upon retraction, particularly, at high ionic strengths (Fig. 2b). Let us now discuss such events in more detail.

Fig. 5 shows additional force profiles, which illustrate the diverse nature of the molecular adhesion events observed. For
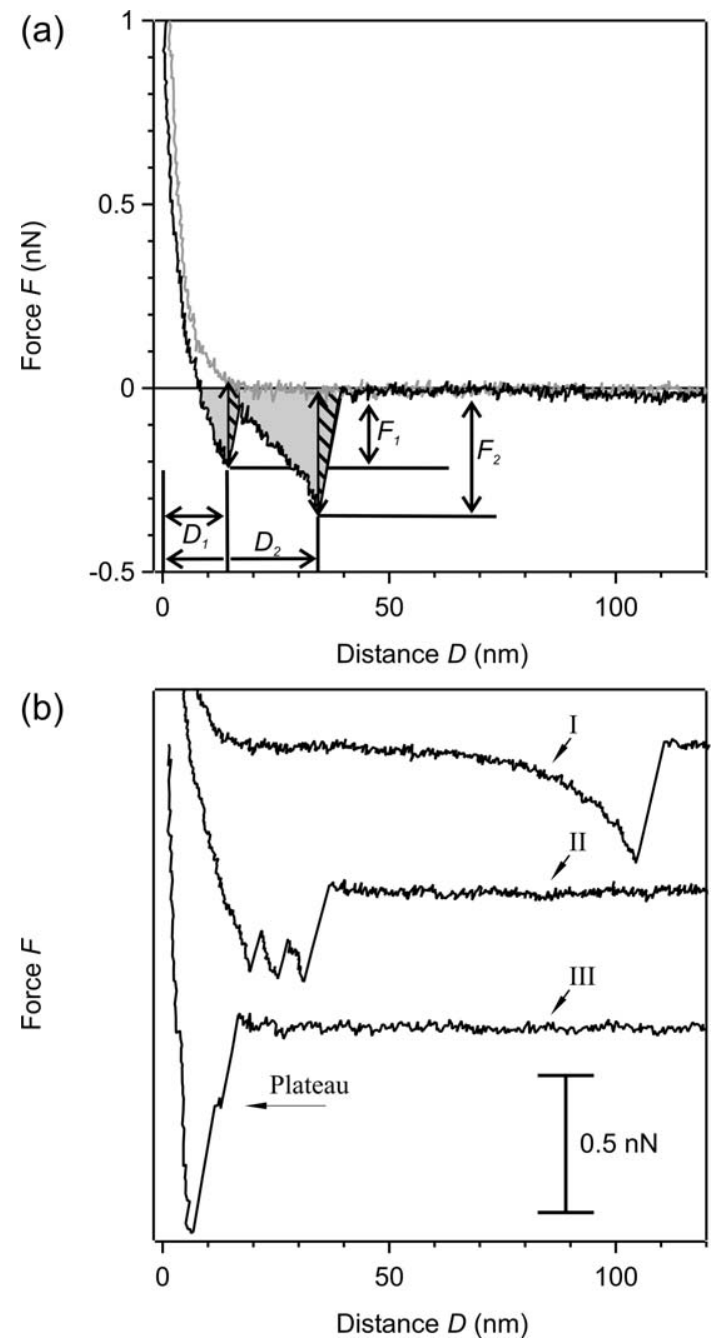

Fig. 5. Representative force profiles between colloidal silica probe and quartz substrate with pre-adsorbed layers of PEI prepared at high salt adsorption conditions illustrating different molecular adhesion events. (a) Two rubber-like events with the corresponding forces and distances measured at $100 \mathrm{mM}$ for $5000 \mathrm{kDa}$ PEI. The area used for calculating the work of adhesion is indicated in grey and the contribution during jump-out is shaded. (b) Additional retraction force profiles, which were displaced vertically for clarity. Single molecule pulling event (I, $5000 \mathrm{kDa}$ and $100 \mathrm{mM}$ ), sequence of erratic events (II, $5000 \mathrm{kDa}$ and $10 \mathrm{mM}$ ), and short-range adhesion event (III, $2 \mathrm{kDa}$ and $100 \mathrm{mM}$ ) showing a plateau due to desorption (arrow).

high molecular mass PEI, multiple adhesion events with several minima are the rule (Figs. $2 \mathrm{c}$ and $5 \mathrm{a}$ ). Occasionally, events with a single minimum are observed (Fig. 5b, curve I), which strongly remind of single molecule pulling events as observed for linear neutral polymers and polyelectrolytes [23,42-45]. It should be pointed out, however, that the present force-extension curves show typically much less curvature than in the case of linear polymers. These events probably originate from the stretching of individual polymer segments, several of which are being extended simultaneously within the branched structure. Simultaneous stretching of these interconnected segments results in rubber-like behavior, and leads to almost linear forceextension relationships (Fig. 5a, second event). Such events occur frequently besides erratic events (Fig. 5b, curve II). The latter are probably just a succession of several short rubber- 

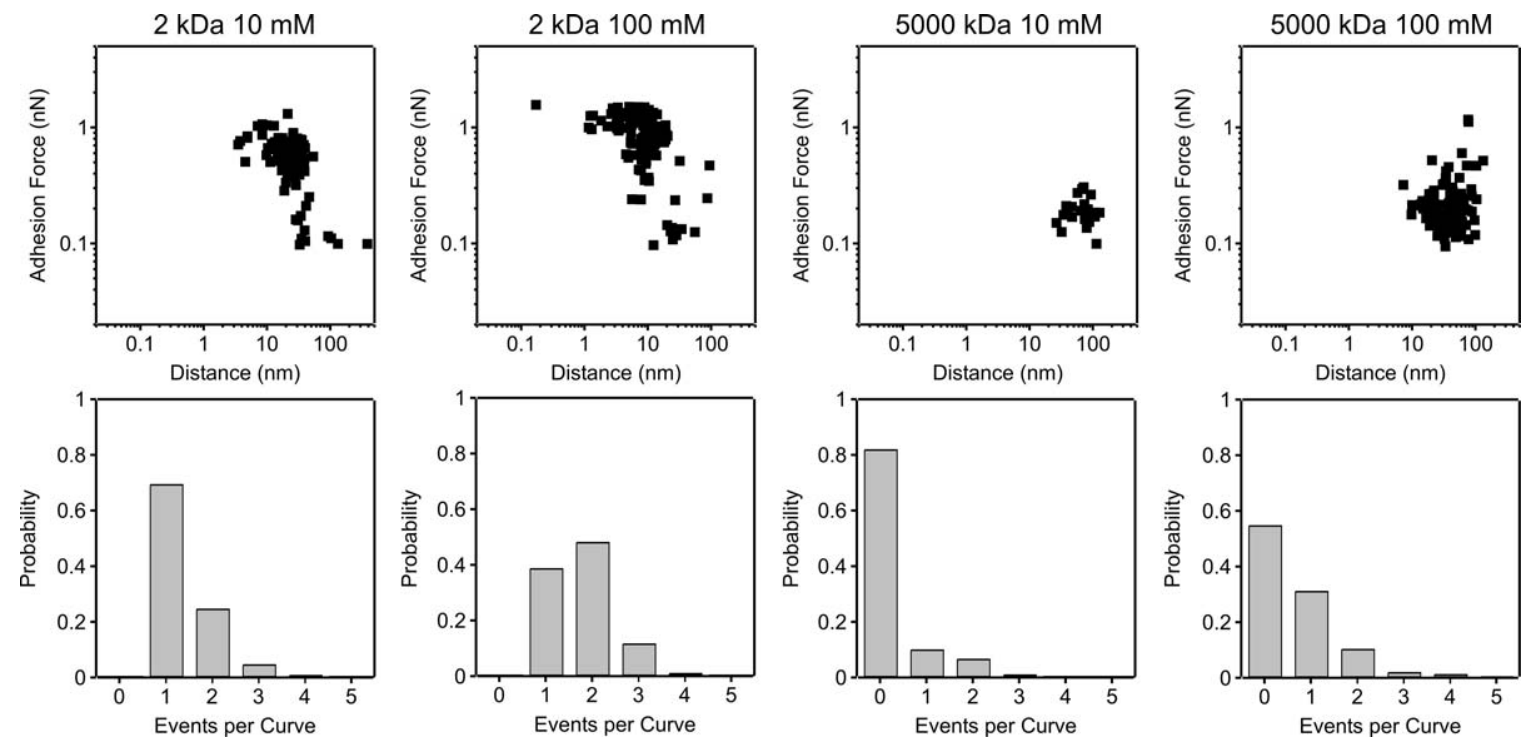

Fig. 6. Molecular adhesion events between pre-adsorbed PEI layers on silica prepared at high salt adsorption conditions and investigated at $\mathrm{pH} 4$ and different ionic strengths in $\mathrm{KCl}$. (Top) Scatter plot of the desorption force as a function of the corresponding distance. (Bottom) Probability distribution of the number of events per curve. The columns correspond to the molecular masses of 2 and $5000 \mathrm{kDa}$ at ionic strengths of 10 and $100 \mathrm{mM}$.

like events, which remain unresolved given the noise of our experimental setup. In some cases, desorption events are observed, which feature one or several force plateaus (Fig. 5b, curve III). These plateaus resemble those found for linear polyelectrolytes [43], but they are much shorter in the present case, and hardly exceed a few nanometers. These desorption events occur most frequently for the low molecular mass PEI, which equally exhibits an unspecific adhesion minimum at small distances $(<10 \mathrm{~nm}$, Fig. 5b, curve III) directly after separation. While no molecular adhesion events are observed at the lowest ionic strength of $0.1 \mathrm{mM}$, they occur frequently at high ionic strengths. The adhesion events occur at larger distances, and with the exception of the $2 \mathrm{kDa}$ sample, no unspecific adhesion minima at short distances are observed.

Analyzing these molecular adhesion events quantitatively is difficult due to their rich structure and random character. The most marked feature is the jump-off of the cantilever, which is accompanied with a local minimum in the force. Two of these events are indicated in Fig. 5a, occurring at the rupture distances $D_{1}$ and $D_{2}$ with the corresponding maximal magnitude of the forces $F_{1}$ and $F_{2}$, referred to as pull-off forces. All molecular adhesion events are analyzed with respect to the rupture distance and the pull-off force. The results are summarized in Fig. 6 and Table 2, where low molecular mass PEI (two left columns) is compared with high molecular mass PEI (two right columns).

Scatter plots of the pull-off forces as a function of the distance are given in Fig. 6a. For low molecular mass PEI, one observes events at separation distances of few tenths of nanometers and maximum pull-off forces above one $\mathrm{nN}$. For high molecular mass PEI, on the other hand, the events extend beyond $100 \mathrm{~nm}$, but the forces are centered around $0.3 \mathrm{nN}$. The average distances and pull-off forces reported in Table 2 also reflect these trends. At an ionic strength of $10 \mathrm{mM}$, we have an average distance of $28 \mathrm{~nm}$ and average pull-off forces of
Table 2

Typical characteristics of molecular adhesion events

\begin{tabular}{lllll}
\hline Molecular mass & $2 \mathrm{kDa}$ & & $5000 \mathrm{kDa}$ & \\
\hline Ionic strength $I(\mathrm{mM})$ & 10 & 100 & 10 & 100 \\
Mean events per curve & 1.4 & 1.8 & 0.3 & 0.7 \\
Probability of no event & 0 & 0 & 0.82 & 0.56 \\
Mean force $(\mathrm{nN})$ & 0.58 & 0.9 & 0.19 & 0.25 \\
Mean distance $(\mathrm{nm})$ & 28 & 12 & 71 & 43 \\
Mean work $\left(\times 10^{-18} \mathrm{~J}\right)$ & 7 & 12 & 0.8 & 1.7 \\
\hline
\end{tabular}

$0.58 \mathrm{nN}$ for low molecular mass PEI, while for high molecular mass PEI these numbers are $71 \mathrm{~nm}$ and $0.19 \mathrm{nN}$. The rupture distances reported here are somewhat larger, but comparable to the lateral dimensions of adsorbed PEI molecules [40].

In general, the adhesion data are not as well reproducible as the long-range interaction forces, particularly, at intermediate ionic strengths. These difficulties can be partially attributed to sampling errors caused by the broad distributions encountered, and limited number of molecular adhesion events observed (typically 50-200). Furthermore, the adhesion on the single molecule level appears to be sensitive to the polyelectrolyte conformation in the adsorbed state, which might be time dependent and vary between the different preparations to some extent.

Nevertheless, the following trends can be established with confidence. Few or no molecular adhesion events are observed at low ionic strengths, while at high ionic strength these events are frequent. We explain this trend by the more effective screening of the repulsive interactions between the PEI molecules at higher ionic strengths due to the reduced Debye length (cf. Eq. (1)). These reduced interactions result in an increased probability of interpenetration of the PEI molecules upon approach and promote their consecutive adsorption at uncovered patches on the other surface. At low ionic strength, there is lit- 
tle interpenetration of the layers and thus no adhesion events are observed.

The mean distances at which these events occur, increase with increasing ionic strength and with increasing molecular mass. The mean force of these events also increases with the ionic strength, but decreases with the molecular mass. Evidently, larger PEI molecules can be pulled further than smaller ones. On the other hand, the extension forces are lower since the large molecules are more flexible and less well anchored to the surface. These trends are rather well obeyed for all molecular masses and both adsorption conditions, with the exception of the $30 \mathrm{kDa}$ fraction, where no adhesion is observed at ionic strengths $\leqslant 10 \mathrm{mM}$. Differences in the degree of branching or in the sample fractionation procedure could represent the reasons for this unusual behavior.

Given the complicated structure of the retraction curves, the scatter plots as well as the number of events quantify the adhesion between PEI-coated surfaces only in approximate manner. For this reason, we have further estimated the work that is necessary to separate the surfaces. This work of adhesion corresponds to the area under the force curve upon retraction and can be obtained by its numerical integration (see Fig. 5a). The difficulty of this approach is that during the jump-off instabilities, the slope of force-distance curve reflects simply the force constant of the cantilever, but does not correspond to the actual retraction force profile. However, the contribution of these instabilities (shaded area, Fig. 5a) to the adhesion work is relatively small, when compared to the other contributions (grey area, Fig. 5a). This contribution is typically $<20 \%$, and only a small error will be introduced. The only exception was the PEI of $2 \mathrm{kDa}$, where the contribution of the instability of the shortrange adhesion cannot be neglected. Thus, the estimated work of adhesion must be viewed with caution for PEI of $2 \mathrm{kDa}$.

The mean work of adhesion per each retraction was $1.7 \times$ $10^{-18} \mathrm{~J}$ for PEI of $5000 \mathrm{kDa}$ at an ionic strength of $100 \mathrm{mM}$ (Table 2). At $10 \mathrm{mM}$ the work was $0.8 \times 10^{-18} \mathrm{~J}$, and this value decreases with the decreasing ionic strength. This decrease of the work of adhesion with decreasing ionic strength could be confirmed for all molecular masses. At $100 \mathrm{mM}$ we have observed a mean work of adhesion of $(6 \pm 2) \times 10^{-18} \mathrm{~J}$ for all samples and preparations, while at $0.1 \mathrm{mM}$ this work was $<10^{-19} \mathrm{~J}$. At intermediate ionic strengths, the data are less reproducible. The mean work of adhesion shows no clear trend with the molecular mass and is very similar for the two different adsorption conditions. The strong dependence on the ionic strength of the adhesion can be explained through the fact that the bridging between the surfaces necessitates the availability of free substrate patches on the other side. The accessibility of these patches is facilitated at high ionic strength through the reduced electrostatic repulsion between the polymer segments or eventually partial polymer desorption, leading to more free patches on the substrate where polymer from the other surface may adsorb.

In order to compare the work of adhesion with values available in the literature [8,17], the work of adhesion obtained from the force curves has to be normalized to the unit area. In difference to the SFA, the necessary contact area cannot be measured directly with the colloidal probe, but a rough estimation can be made. Let us first consider the situation of two fully compressible, infinitely soft PEI-layers with a thickness $l$ in contact. The contact area is then defined by the overlap of the polymer layers and represents an approximate upper limit of the area in which polymer segments can bridge directly to the other surface. The radius $r$ of the outer rim of the circular overlap region in the sphere-plane geometry is obtained from a simple geometrical argument. In this approximation, the spherical colloidal probe of radius $R$ and the planar substrate touch at the apex point of the sphere and of the rim is given by $r=\sqrt{4 R l}$. With a typical radius $R$ of the colloidal probe of $3.4 \mu \mathrm{m}$ and a layer thickness $l$ of $4 \pm 2 \mathrm{~nm}$, we obtain contact radii of the order of $230 \pm 60 \mathrm{~nm}$.

This approximation of fully compressible layers introduces several assumptions. Two are the most important ones. Firstly, the approximation neglects the deformation of the silica and quartz surfaces. For bare silica surfaces, the radius of the circular contact area can be estimated by classical Hertz theory [36], and is on the order of $10 \mathrm{~nm}$ for a typical loading force of $10 \mathrm{nN}$ and a bulk Young modulus of $72 \mathrm{GPa}$ [46]. For this reason, this contribution can be neglected. Secondly, this approximation neglects the finite thickness of the PEI-layer upon compression, which was measured by SFA to be on the order of $2 \mathrm{~nm}$ [16]. For substantially thinner adsorbed layers, such as lipids, the elastic properties are given primarily by the underlying substrate $[47,48]$. On the other hand, for an infinitely thick polymer layer with a bulk Young modulus of $10 \mathrm{MPa}$, the Hertz theory predicts a contact radius of $150 \mathrm{~nm}$. This value for the Young modulus is in the upper range of values reported for gels and polymer layers [49]. The contact radius estimated in this fashion represents an upper limit, and for this reason the estimate given by the simple geometrical model should be appropriate. Nevertheless, it should be emphasized that the elastic properties of thin, soft polymer films on solid substrates are influenced by the substrate [50], and Hertz theory is inappropriate to describe the deformation of such a layer.

Taking the typical work of adhesion of $6 \times 10^{-18} \mathrm{~J}$, obtained from the bridging events at $100 \mathrm{mM} \mathrm{KCl}$ as discussed above, and a contact area of radius $230 \pm 60 \mathrm{~nm}$, as estimated by the simple model above, we obtain a work of adhesion per unit area of $0.04 \pm 0.01 \mathrm{mN} / \mathrm{m}$. These values can be compared to the work of adhesion $W$ obtained from the unspecific adhesion force $F_{\text {adh }}$ of $0.9 \mathrm{nN}$ (cf. Table 2) observed for the low molecular mass PEI $(2 \mathrm{kDa})$ at the same ionic strength. From the classical theory of Johnson, Kendall, and Roberts (JKR) [36] we estimate in this case from the relation $F_{\text {adh }}=(3 / 2) \pi R W$, a work of adhesion per unit area $W$ of $0.06 \mathrm{mN} / \mathrm{m}$.

Since these two estimated values for the work of adhesion per unit area agree reasonably well within the uncertainties of our approximation, we suspect that a similar adhesion mechanism is operating for all molecular masses, but the individual adhesion events cannot be resolved for the PEI of lowest molecular mass. This molecule consists of less than 100 monomers and cannot be stretched very much. However, the steric repulsion it generates is similar to the higher molecular mass PEI, both in magnitude and in range. One may thus hypothesize that the magnitude of the electrostatic repulsion between the seg- 
ments as well as the availability of free surface patches might be similar, even though the lateral length scales differ substantially.

The presently observed values for the work of adhesion per unit area of $0.04 \pm 0.01 \mathrm{mN} / \mathrm{m}$ for PEI of all molecular masses should be compared with somewhat lower values around $0.01 \mathrm{mN} / \mathrm{m}$ based on earlier colloidal probe measurements [8]. On the other hand, the MASIF technique results in substantially larger values in the range of $10-30 \mathrm{mN} / \mathrm{m}[16,17]$. However, one must realize that we provided an upper estimate for the contact area and that the latter technique uses much larger radii of curvature, which result in much larger contact areas. Inhomogeneities in the film structure may result in the contact of PEI with patches of bare substrate and would contribute more strongly to the adhesion in the latter case. Moreover, these measurements were carried out at lower salt levels and higher $\mathrm{pH}$, and therefore cannot be compared directly. Nevertheless, the discrepancies indicate that adhesive properties of PEI vary strongly, not only as a function of the solution composition, but also through the detailed preparation history of the sample. The history dependence is probably related to our difficulties to accurately reproduce the molecular adhesion events in the intermediate ionic strength range.

\section{Conclusions}

Interaction forces between pre-adsorbed layers of poly(ethylene imine) (PEI) of different molecular mass have been examined with the colloidal probe technique. During approach, the purely repulsive forces are mainly of electrostatic nature and originate from to the overlap of diffuse layers. The forces can be interpreted quantitatively down to distances of a few nanometers within the PB framework, provided charge regulation is being considered. Close to contact, the forces remain repulsive, probably due to electro-steric interactions between the PEI layers with a range of $2-3 \mathrm{~nm}$. The forces upon approach are almost independent of molecular mass. During retraction, erratic attractive bridging forces are observed originating from molecular adhesion events, which are dominated by rubber-like adhesion. Single-molecule pulling events are less frequently noticed. This behavior is very different from linear polymers, which are normally dominated by pulling events of individual chains. The presently reported behavior is clearly related to the strongly branched architecture of the PEI. With increasing the ionic strength, one observes an increase in the frequency of the molecular adhesion events and the work of adhesion. Strong adhesion forces at short distances are frequent for low molecular mass PEI, while for high molecular mass weaker adhesion forces at larger distances are the rule.

\section{Acknowledgments}

This work was supported by the program TopNano21 administered by the Swiss Commission of Technology and Innovation, BASF Aktiengesellschaft, Ludwigshafen, and the Swiss National Science Foundation.

\section{References}

[1] D. Horn, in: E.J. Goethals (Ed.), Polymeric Amines and Ammonium Salts, Pergamon, Oxford/New York, 1980, p. 333.

[2] G.M. Lindquist, R.A. Stratton, J. Colloid Interface Sci. 55 (1976) 45.

[3] M. Borkovec, S.H. Behrens, in: A. Hubbard (Ed.), Encyclopedia of Colloid and Surface Science, Dekker, New York, 2002, p. 4795.

[4] S. Leporatti, R. Sczech, H. Riegler, S. Bruzzano, J. Storsberg, F. Loth, W. Jaeger, A. Laschewsky, S. Eichhorn, E. Donath, J. Colloid Interface Sci. 281 (2005) 101.

[5] G. Decher, Science 277 (1997) 1232.

[6] M. Schonhoff, J. Phys. Condens. Matter 15 (2003) R1781.

[7] G. Decher, J.D. Hong, J. Schmitt, Thin Solid Films 210 (1992) 831.

[8] V. Bosio, F. Dubreuil, G. Bogdanovic, A. Fery, Colloids Surf. A 243 (2004) 147.

[9] G.B. Sukhorukov, E. Donath, H. Lichtenfeld, E. Knippel, M. Knippel, A. Budde, H. Mohwald, Colloid Surf. A 137 (1998) 253.

[10] A. Dietrich, A. Neubrand, J. Am. Ceram. Soc. 84 (2001) 806.

[11] R. Meszaros, L. Thompson, M. Bos, P. de Groot, Langmuir 18 (2002) 6164.

[12] F. Bouyer, A. Robben, W.L. Yu, M. Borkovec, Langmuir 17 (2001) 5225.

[13] R. Meszaros, I. Varga, T. Gilanyi, Langmuir 20 (2004) 5026.

[14] I.M. Nnebe, R.D. Tilton, J.W. Schneider, J. Colloid Interface Sci. 276 (2004) 306

[15] P.M. Claesson, A. Dedinaite, O.J. Rojas, Adv. Colloid Interface Sci. 104 (2003) 53.

[16] P.M. Claesson, O.E.H. Paulson, E. Blomberg, E.H. Burns, Colloids Surf. A $123-124$ (1997) 341.

[17] E. Poptoshev, P.M. Claesson, Langmuir 18 (2002) 2590.

[18] K. Lowack, C.A. Helm, Macromolecules 31 (1998) 823.

[19] W.A. Ducker, T.J. Senden, R.M. Pashley, Nature 353 (1991) 239.

[20] H.J. Butt, Biophys. J. 60 (1991) 1438.

[21] P.G. Hartley, I. Larson, P.J. Scales, Langmuir 13 (1997) 2207.

[22] S. Biggs, Langmuir 11 (1995) 156.

[23] G.J.C. Braithwaite, A. Howe, P.F. Luckham, Langmuir 12 (1996) 4224.

[24] T.J. Senden, J.M. di Meglio, P. Auroy, Eur. Phys. J. B 3 (1998) 211.

[25] G.X. Sun, H.J. Butt, Macromolecules 37 (2004) 6086.

[26] G.B. Sukhorukov, H. Mohwald, G. Decher, Y.M. Lvov, Thin Solid Films 285 (1996) 220.

[27] K. Buscher, K. Graf, H. Ahrens, C.A. Helm, Langmuir 18 (2002) 3585.

[28] U.Y. Wang, M. Schonhoff, H. Mohwald, J. Phys. Chem. B 108 (2004) 4767.

[29] M. Borkovec, G.J.M. Koper, Macromolecules 30 (1997) 2151.

[30] W. Kern, D.A. Puotinen, RCA Rev. 31 (1970) 187.

[31] J.L. Hutter, J. Bechhoefer, Rev. Sci. Instrum. 64 (1993) 1868.

[32] H.J. Butt, M. Jaschke, Nanotechnology 6 (1995) 1.

[33] J.E. Sader, J.W.M. Chon, P. Mulvaney, Rev. Sci. Instrum. 70 (1999) 3967.

[34] B. Cappella, G. Dietler, Surf. Sci. Rep. 34 (1999) 1.

[35] H.J. Butt, in: A.J. Bard, M. Stratmann (Eds.), Encyclopedia of Electrochemistry, Wiley-VCH, Weinheim, 2003, p. 225.

[36] J. Israelachvili, Intermolecular and Surface Forces, Academic Press, London, 1992.

[37] R. Pericet-Camara, G. Papastavrou, S.H. Behrens, M. Borkovec, J. Phys. Chem. B 108 (2004) 19467.

[38] P.G. Hartley, P.J. Scales, Langmuir 14 (1998) 6948.

[39] C. Gergely, B. Senger, J.C. Voegel, J.K.H. Horber, P. Schaaf, J. Hemmerle, Ultramicroscopy 87 (2001) 67.

[40] A. Pfau, W. Schrepp, D. Horn, Langmuir 15 (1999) 3219.

[41] S.H. Behrens, M. Borkovec, J. Phys. Chem. B 103 (1999) 2918.

[42] C. Ortiz, G. Hadziioannou, Macromolecules 32 (1999) 780.

[43] T. Hugel, M. Grosholz, H. Clausen-Schaumann, A. Pfau, H. Gaub, M. Seitz, Macromolecules 34 (2001) 1039.

[44] M. Rief, F. Oesterhelt, B. Heymann, H.E. Gaub, Science 275 (1997) 1295.

[45] W.K. Zhang, S. Zou, C. Wang, X. Zhang, J. Phys. Chem. B 104 (2000) 10258.

[46] R.C. Weast, M.J. Astle, CRC Handbook of Chemistry and Physics, 60th ed., CRC Press, Boca Raton, FL, 1980. 
[47] Y.L. Chen, C.A. Helm, J.N. Israelachvili, J. Phys. Chem. 95 (1991) 10736.

[48] Y.L. Chen, C.A. Helm, J.N. Israelachvili, Langmuir 7 (1991) 2694.
[49] L. Richert, A.J. Engler, D.E. Discher, C. Picart, Biomacromolecules 5 (2004) 1908.

[50] J. Domke, M. Radmacher, Langmuir 14 (1998) 3320. 